\title{
Ab-Initio Surface Hopping and Multiphoton Ionisation Study of the Photodissociation Dynamics of $\mathrm{CS}_{2}$
}

\author{
Darren Bellshaw, ${ }^{a}$ Daniel A. Horke,${ }^{b, c}$ Adam D. Smith,${ }^{d}$ Hannah M. \\ Watts, ${ }^{d}$ Edward Jager, ${ }^{d}$ Emma Springate, ${ }^{e}$ Oliver Alexander,${ }^{e}$ Cephise \\ Cacho, ${ }^{e}$ Richard T. Chapman, ${ }^{e}$, Adam Kirrander, ${ }^{a}$ and Russell S. Minns ${ }^{d}$ \\ ${ }^{a}$ EaStCHEM, School of Chemistry, University of Edinburgh, David Brewster Road, \\ Edinburgh EH9 3FJ, United Kingdom. \\ ${ }^{b}$ Center for Free-Electron Laser Science, DESY, Notkestrasse 85, 22607 Hamburg, \\ Germany \\ ${ }^{c}$ The Hamburg Centre for Ultrafast Imaging, University of Hamburg, Luruper Chaussee \\ 149, 22761 Hamburg, Germany \\ ${ }^{d}$ Chemistry, University of Southampton, Highfield, Southampton SO17 1BJ, UK \\ ${ }^{e}$ Central Laser Facility, STFC Rutherford Appleton Laboratory, Didcot, Oxfordshire \\ $O X 110 Q X, U K$
}

\begin{abstract}
New ab-initio surface hopping simulations of the excited state dynamics of $\mathrm{CS}_{2}$ including spin-orbit coupling are compared to new experimental measurements using a multiphoton ionisation probe in a photoelectron spectroscopy experiment. The calculations highlight the importance of the triplet states even in the very early time dynamics of the dissociation process and allow us to unravel the signatures in the experimental spectrum, linking the observed changes to both electronic and nuclear degrees of freedom within the molecule.
\end{abstract}

Keywords: Photodissociation, Photoelectron spectroscopy, Theoretical Chemistry, Non-adiabatic dynamics

\section{Introduction}

2 The dissociation dynamics of $\mathrm{CS}_{2}$ following $\mathrm{UV}$ excitation have been 3 a benchmark in chemical dynamics for many years, with numerous exper4 imental studies in both the time and frequency domain, see for example $5[1,2,3,4,5,6,7,8,9,10]$. This lasting fascination with $\mathrm{CS}_{2}$ can be traced 
to the efficient dissociation, dictated by complex dynamics on multiple coupled electronic states. Despite intense experimental study, the fast dynamics and the high ionisation limits of intermediates and final products have limited the experimental view to specific points along the full dissociation path, such that open questions remain even for this structurally simple molecule. The origin of the complexity derives from the near degeneracy of the optically bright ${ }^{1} \mathrm{~B}_{2}\left({ }^{1} \Sigma_{u}^{+}\right)$state with multiple other electronic states at linear geometry, which leads to highly efficient population transfer and strongly coupled multistate dynamics. The mixing of the electronic states leads to dissociation and the formation of a ground state CS $\left(\mathrm{X}^{1} \Sigma^{+}\right)$molecule in conjunction with atomic sulphur in either the spin forbidden ground state, ${ }^{3} \mathrm{P}$, or a spin allowed excited state, ${ }^{1} \mathrm{D}$. While the exact branching ratio has proven difficult to define accurately, the spin forbidden product is seen to dominate in most experimental studies [11, 2, 12], highlighting the importance of spin-orbit coupling for an accurate description. Considering how well studied this molecule has been experimentally, calculations of the dynamics have been limited with, as far as we are aware, no simulations accounting for the spin-orbit coupling that drives the dominant dissociation process. In this work we combine ab-initio surface hopping simulations of the dissociation dynamics of $\mathrm{CS}_{2}$ with new photoelectron spectroscopy measurements using a multiphoton probe to study the effect of spin-orbit coupling on the early time dynamics of the molecule.

Previous dynamics calculations have focused on the singlet state dynamics and the effect of non-adiabatic coupling on measured photoelectron angular distributions obtained following excitation at $201 \mathrm{~nm}$ and ionisation with 268 $\mathrm{nm}$ [13]. These calculations provide a very good measure of the photoangular distribution, which they claim suggests that the lack of spin-orbit coupling in the model does not affect the calculated early time dynamics. It should be noted that the rather low energy probe used in the experiment means that only the singlet states could be ionised and that any effect of the triplet in the angular distributions would not be observable. The measurements and theory therefore do not take into account the population transfer between the initially excited singlet manifold and the accessible triplet states. This point is highlighted by recent time-resolved VUV photoelectron spectroscopy experiments by Spesyvtsev et al. [4] using a 20 fs $159 \mathrm{~nm}(7.8 \mathrm{eV})$ probe pulse. These experiments provide the most detailed maps of the excited state dynamics to date, and show large changes in electron kinetic energy as the molecule undergoes bending vibrations with an almost $3 \mathrm{eV}$ shift in the 
measured electron kinetic energy in 40 fs. The probed dynamics occur on the singlet surfaces and, as the molecule continues to vibrate, the population is transferred into lower lying electronic states which are outside the observation window provided even by their VUV probe.

\section{Theory}

\subsection{Computational methods}

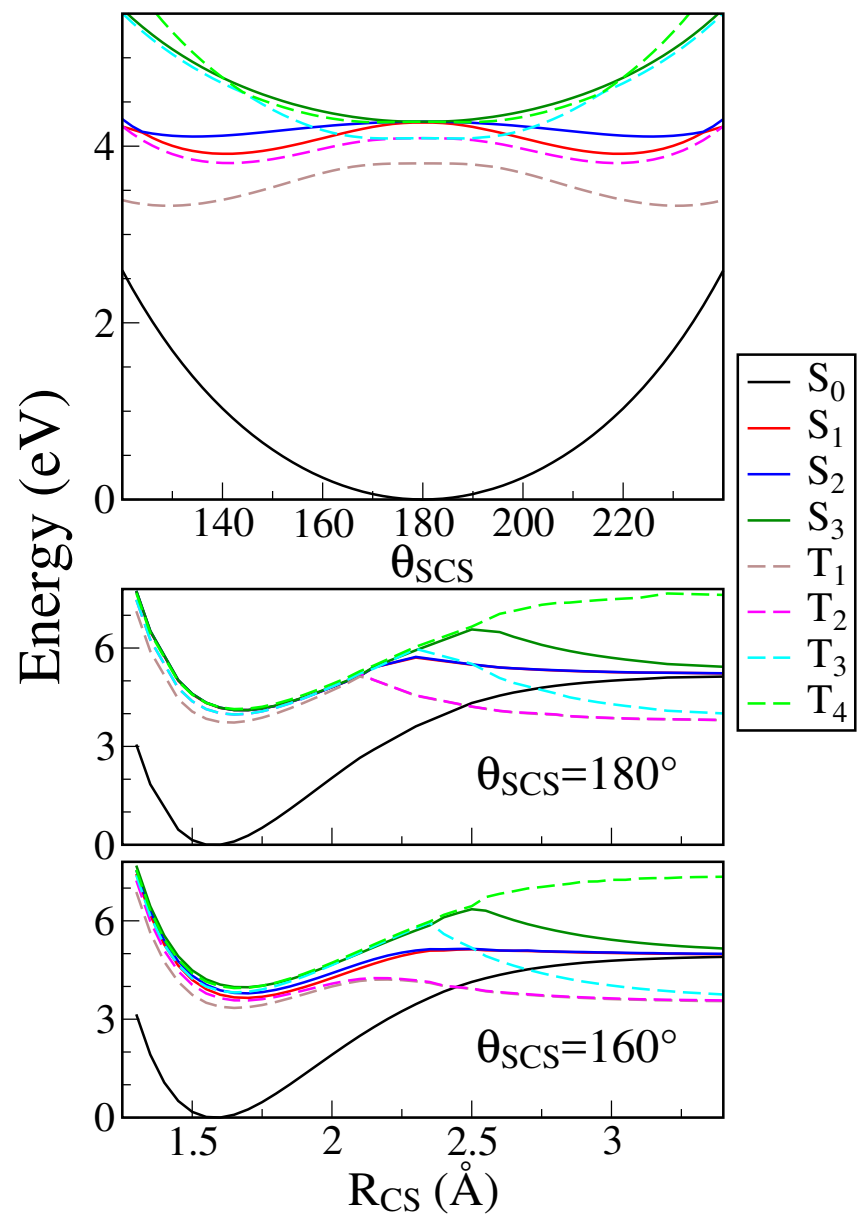

Figure 1: Bending $\theta_{\mathrm{SCS}}$ (top), and radial asymmetric stretch $R_{\mathrm{CS}}$ for linear $\theta_{\mathrm{SCS}}=180^{\circ}$ (middle) and bent $\theta_{\mathrm{SCS}}=160^{\circ}$ (bottom), potential energy curves calculated at the SA8CAS $(16,12)$ /aug-cc-pvTZ level for the first four singlet and triplet states of $\mathrm{CS}_{2}$. Remaining degrees of freedom are frozen at their equilibrium values. 
Ab-initio electronic structure calculations were performed using the Molpro 2015.1 [14] suite of programs at the CAS(16,12)/aug-cc-pvTZ and augcc-pvQZ level of theory using the full valence $(16,12)$ active space with bonding and antibonding $\sigma$ and $\pi$ molecular orbitals and sulfur atom lone pairs. Ground state geometry optimisation using $\operatorname{CAS}(16,12) /$ aug-cc-pvQZ resulted in $R_{\mathrm{CS}}=1.569 \AA$ and $\theta_{\mathrm{SCS}}=0^{\circ}$. Angular and radial cuts through the singlet and triplet potential energy surfaces are shown in Fig. 1, and vertical excitation energies and oscillator strengths for the first four excited singlet states are given in Table 1. The potential energy curves in Fig. 1 are broadly in keeping with previous ab-initio calculations $[15,16,17,18,19]$.

Table 1: Vertical excitation energies $\left(\triangle E=E\left(\mathrm{~S}_{i}\right)-E\left(\mathrm{~S}_{0}\right)\right)$ and oscillator strengths from the ground state to the first four excited singlet states of $\mathrm{CS}_{2}$, calculated using SA5CAS(16,12)-SCF/aug-cc-pvQZ with CASPT2 corrections to the energies. The excitation energies are calculated at the equilibrium geometry $\left(\theta_{\mathrm{SCS}}=180^{\circ}\right.$ and $\left.R_{\mathrm{CS}}=1.569 \AA\right)$, while oscillator strengths are calculated at $\theta_{\mathrm{SCS}}=160^{\circ}$ since transition are very weak in the linear geometry.

\begin{tabular}{|l|c|c|}
\hline State & Energy (eV) & Oscillator strength \\
\hline $\mathrm{S}_{1}$ & 3.821 & 0 \\
$\mathrm{~S}_{2}$ & 3.836 & 0.004282 \\
$\mathrm{~S}_{3}$ & 3.836 & 0 \\
$\mathrm{~S}_{4}$ & 6.430 & 0.000834 \\
\hline
\end{tabular}

We simulate the dynamics of photoexcited $\mathrm{CS}_{2}$ using the code SHARC $[20,21]$ interfaced with MOLPRO [14]. SHARC treats nuclear motion classically, but nonadiabatic effects and spin-orbit coupling [22] are included using the fewest-switches surface-hopping approach [23]. The spin-orbit coupling is treated in the diabatic representation and has been shown to replicate branching dynamics in IBr when compared to full quantum dynamics simulations [22]. In contrast to previous singlet-only simulations [13], we propagate the dynamics on the four lowest singlet and triplet electronic states. To keep the simulations computationally feasible, we perform the electronic structure calculations at the SA8-CAS $(8,6)-\mathrm{SCF} / 6-31 \mathrm{G}^{*}$ level, which qualitatively reproduces the potential energy curves shown in Fig. 1. The differences to the CAS(16,12)/aug-cc-pvTZ level calculations are minor at small and large bond-lengths, but at intermediate distances the smaller active space gives rise to elevated barriers to dissociation, that lead to transient trapping of population in the T2 state (see discussion in Section 2.2). Initial positions are 


\subsection{Computational results}

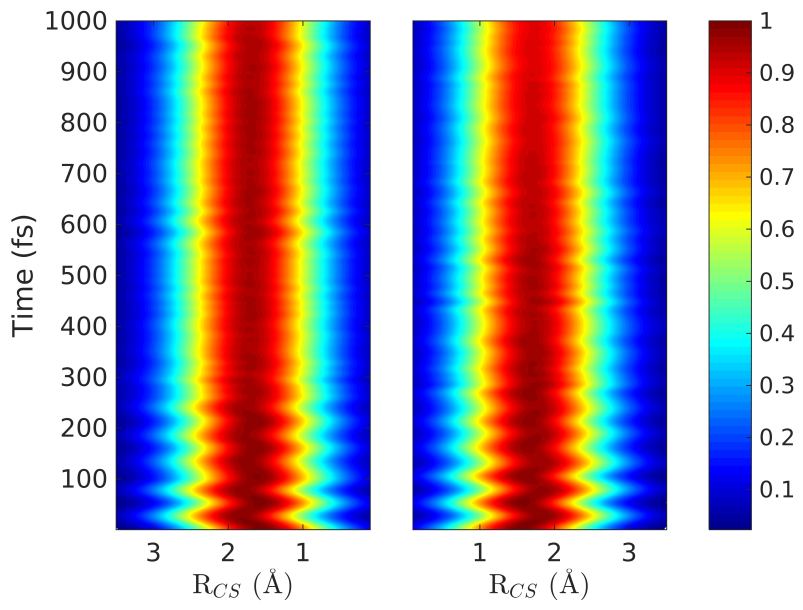

(a) Bondlengths $R_{\mathrm{CS}}$ as a function of time.

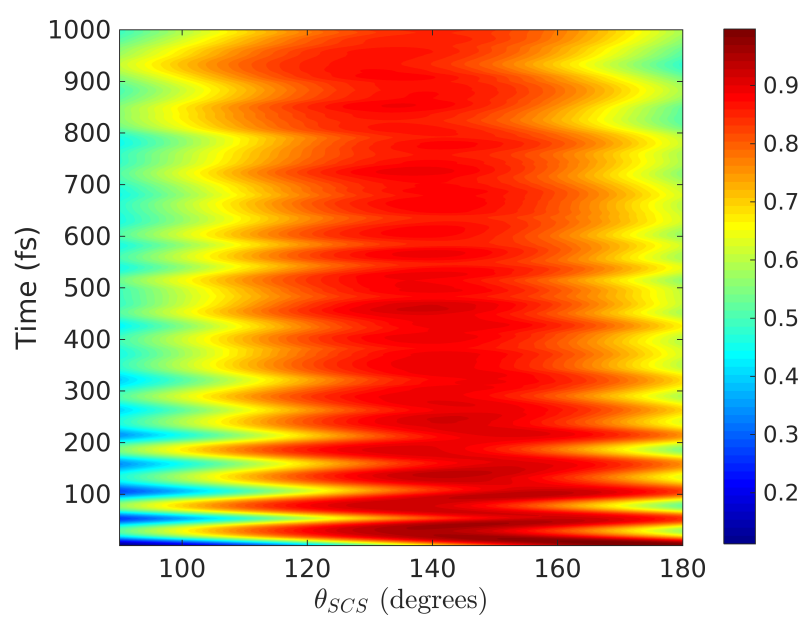

(b) Bending angle $\theta_{\mathrm{SCS}}$ as a function of time.

Figure 2: Probability density evolution of the $\mathrm{CS}_{2}$ geometry in terms of the two bondlengths $R_{\mathrm{CS}}$ (Fig. 2a) and bending angle $\theta_{\mathrm{SCS}}$ (Fig. 2b) from the simulations.

generated from a Wigner distribution based on the $\mathrm{CAS}(8,6) / 6-31 \mathrm{G}^{*}$ ground state vibrational frequencies and the oscillator strength of each geometry, and kinetic energy is assigned based on the required excitation energy and the experimental pump pulse energy. Following this protocol, $85 \%$ of trajectories begin in the $B^{1} \mathrm{~B}_{2}$ state. A total of 369 trajectories are launched, of which 197 reach 500 fs and 114 reach $1000 \mathrm{fs}$, using a time step of 0.5 fs. The reasons that some trajectories fail to reach 1000 fs are related to the electronic structure calculations and include numerical problems such as excessive gradients in the CI or failure in convergence of the MCSCF calculations.

Excitation of $\mathrm{CS}_{2}$ triggers bending and vibrational motion in the molecule, as can be seen in Fig. 2, which shows the probability density evolution of the molecular geometry as a function of the C-S bond-lengths, $R_{\mathrm{CS}}$, and the bending angle, $\theta_{\mathrm{SCS}}$. During the first 100 fs the vibrations are dominated by the symmetric stretch, but at later times energy flows into the asymmetric stretch. The frequencies of vibrations are somewhat over-estimated compared to the experimental values, presumably due to slight differences in the ab-initio potential energy surfaces at the $\operatorname{CAS}(8,6)$ level. The total 


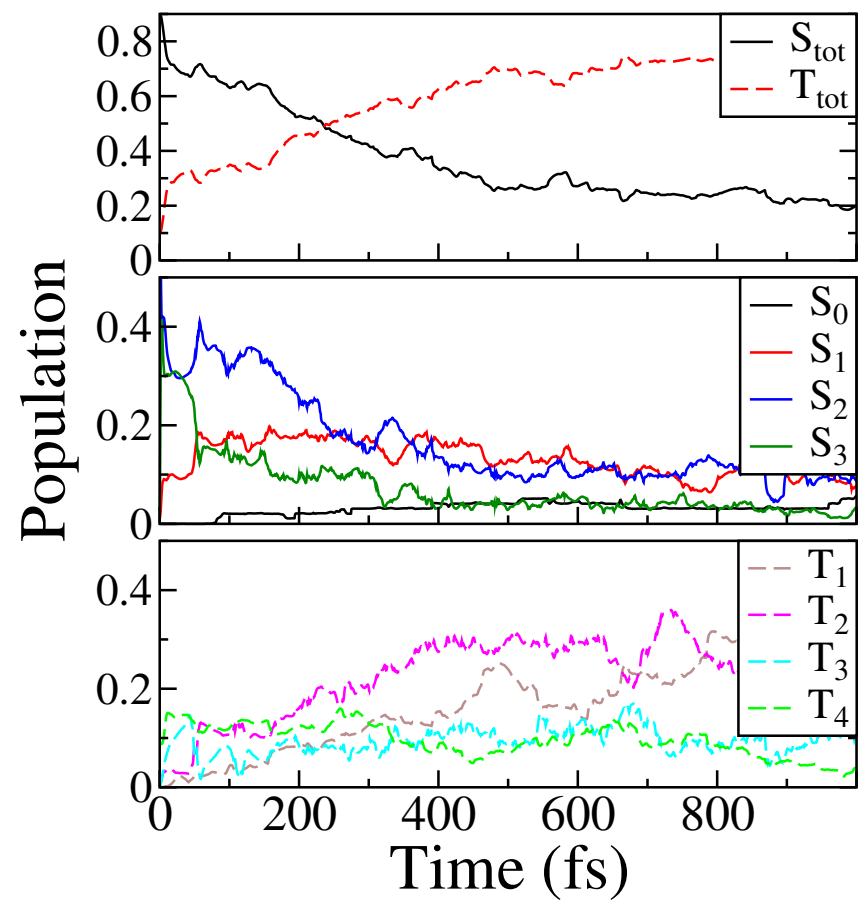

Figure 3: Time-dependent adiabatic state populations from simulation of $\mathrm{CS}_{2}$ dynamics. The top panel shows the total singlet (excluding the $\mathrm{S}_{0}$ ground state) and total triplet populations as a function of time. The middle panel shows the populations for each singlet state and the bottom panel the corresponding data for the triplet states.

fraction of dissociated molecules in the full set of 369 trajectories is $22 \%$, which constitutes a lower bound since only about a quarter of the trajectories reach $1000 \mathrm{fs}$. Dissociation occurs predominantly in the triplet states, with $89 \%$ of the trajectories that dissociate occurring on the triplet surfaces. The lower degree of dissociation compared to the experiments can be traced to the topology of the potential energy surfaces at the level of $a b$-initio theory employed in the simulations, as discussed below.

The electronic state populations as a function of time are shown in Fig. 3. Initial excitation onto the $\mathrm{S}_{2}{ }^{1} \mathrm{~B}_{2}\left({ }^{1} \Sigma_{u}^{+}\right)$state is followed by rapid decay onto the singlet $S_{3}$ and $S_{1}$ potentials, as well as a redistribution of population onto the manifold of triplet states via spin-orbit coupling. The nonadiabatic transfer of population between the singlet states correlates strongly with the bending motion of the molecule, with efficient transfer predominantly occurring close to the linear geometry where states are (near)-degenerate. 
This gives rise to a periodic beating in both the individual singlet state populations and in the total singlet population. Over time there is a build-up of population in $\mathrm{T}_{2}$ at $t>400 \mathrm{fs}$, and a subsequent rise of population in $\mathrm{T}_{1}$ at around $t>800 \mathrm{fs}$, due to population transfer from $\mathrm{T}_{2}$ to $\mathrm{T}_{1}$. The build-up in $\mathrm{T}_{1}$ appears to be an artifact due to the $\mathrm{SA} 8-\mathrm{CAS}(8,6)-\mathrm{SCF} / 6-31 \mathrm{G}^{*}$ ab-initio calculations, which increases the relative barrier height for dissociation on the $\mathrm{T}_{1}$ and $\mathrm{T}_{2}$ potentials by $\approx 1.5 \mathrm{eV}$ (see Section 2.1 ), hindering dissociation and leading to the observed accumulation of population in $\mathrm{T}_{2}$. Consequently, it is reasonable to assume that the population trapped in $\mathrm{T}_{2}$ in actual fact dissociates as observed in the experiment. Nevertheless, despite that the simulations underestimate the amount of $t<1$ ps dissociation via the triplet states, the short-time $t<400$ fs dynamics appears quite reliable.

\section{Experiment}

\subsection{Experimental methods}

The experiment has been described in detail previously [24]. Briefly, an amplified femtosecond laser system (Red Dragon, KM Labs) generates 30 fs pulses of $800 \mathrm{~nm}$ light, with a pulse energy of up to $10 \mathrm{~mJ}$ at a repetition rate of $1 \mathrm{kHz}$. The pump pulse is produced via fourth harmonic generation of the fundamental $(800 \mathrm{~nm}$ ) beam, generating photons at around $200 \mathrm{~nm}$. The $200 \mathrm{~nm}$ beam is produced using standard non-linear optics with sequential second, third and fourth harmonic generation in BBO giving a pulse energy of $\sim 1 \mu \mathrm{J}$. The $400 \mathrm{~nm}$ probe is generated by second harmonic generation of the fundamental laser output, producing approximately $5 \mu \mathrm{J}$ per pulse. The pump and probe beams are reflection focused in a near collinear geometry and cross at the centre of the interaction region of a velocity-map imaging (VMI) spectrometer [25], where they intersect the $\mathrm{CS}_{2}$ molecular beam. The pump and probe beams are both linearly polarised in the plane of the VMI detector, perpendicular to the time-of-flight axis. The molecular beam is generated through the expansion of $5 \% \mathrm{CS}_{2}$ in He at 1 bar through a $1 \mathrm{kHz}$ pulsed nozzle (Amsterdam cantilever [26]) with a $100 \mu \mathrm{m}$ aperture. The resulting expansion passes through a $1 \mathrm{~mm}$ skimmer and enters the interaction region of the spectrometer through a hole in the centre of the repeller plate of the VMI spectrometer. The photoelectron spectra are obtained through polar onion-peeling of the background subtracted images [27]. Although the photoelectron angular distributions are obtained, they show no time dependence and as such are not discussed in the results section. 


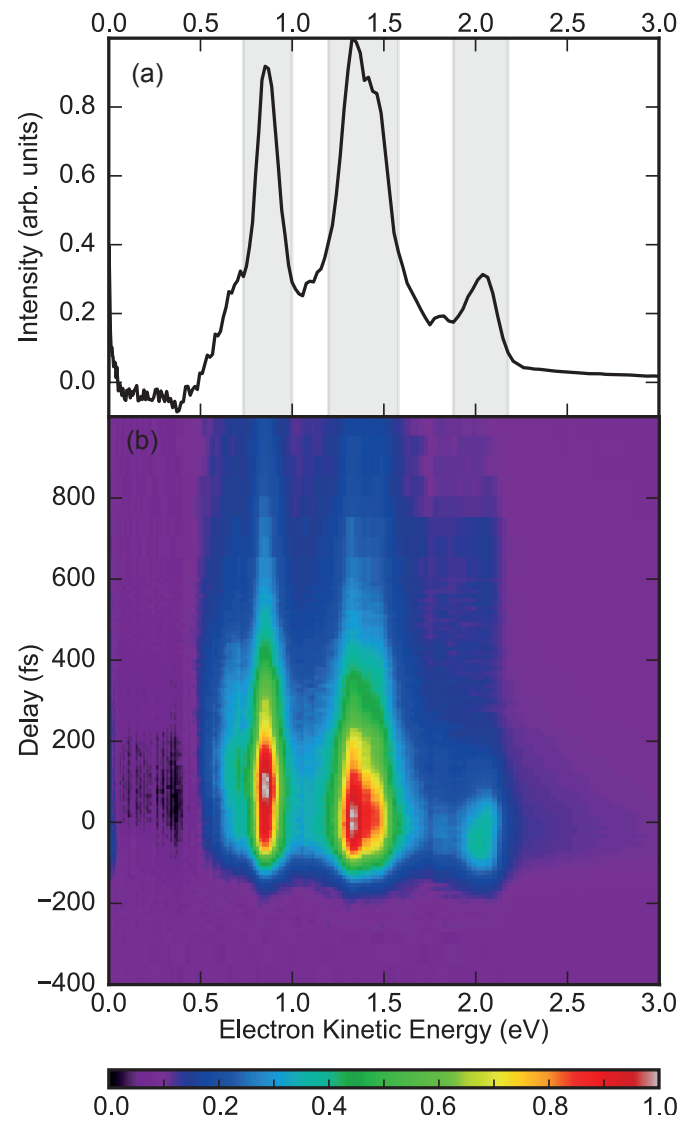

Figure 4: (a) Photoelectron spectrum obtained when the pump and probe pulses are temporally overlapped. The highlighted regions mark those used in the integrated plots shown in figure 5. (b) Photoelectron spectra as a function of pump-probe delay.

\subsection{Experimental results}

The $200 \mathrm{~nm}$ pump excites a vibrational wavepacket, predominantly in the $\mathrm{S}_{2}{ }^{1} \mathrm{~B}_{2}$ excited electronic state. The motion is then probed by non-resonant two-photon absorption at $400 \mathrm{~nm}$. This provides a total energy of $12.5 \mathrm{eV}$, with the ionisation potential of $\mathrm{CS}_{2}$ at $10.07 \mathrm{eV}$. The photoelectron signal obtained when the pump and probe pulse are overlapped in time is plotted in Fig. 4(a) with three main features around $2.1 \mathrm{eV}, 1.4 \mathrm{eV}$ and $0.9 \mathrm{eV}$ electron kinetic energy. The spacing between the features is similar to that seen in previous single-photon ionisation measurements $[1,5]$. The use of a multiphoton probe maintains a clean experimental measurement, without any probe-pump contributions at early times, while maximising the available 


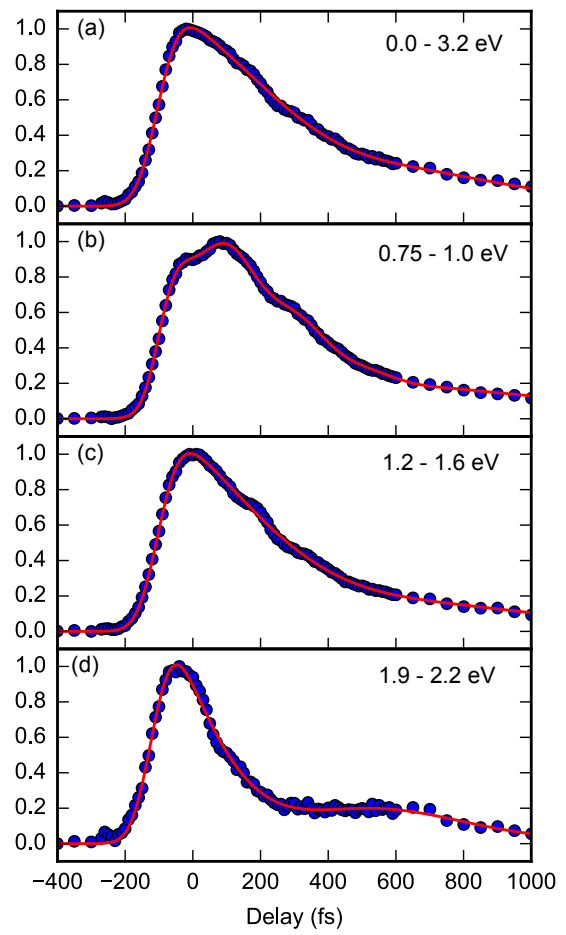

Figure 5: Total integrated photoelectron intensity (a) and intensity within the shaded areas shown in Fig. 4; $0.75-1.00 \mathrm{eV}$ (b), $1.20-1.60 \mathrm{eV}$ (C) and $1.90-2.20 \mathrm{eV}$ (d). Solid lines represent fits to the data. (a,c,d) were fit with a single exponential decay, modulated by a single damped oscillator and convoluted with the instrument response function. However, a second oscillation with a period $\sim 200$ fs is clearly visible in the data. The data in (b) was fit with two damped oscillating components, which reproduce both observed oscillatory features well.

energy for ionisation, such that we can observe much of the initial excited state dynamics.

The time-dependence of the photoelectron spectrum is shown in Fig. 4(b). The three peaks in the spectrum have different appearance times, with those at lower electron kinetic energy appearing after those at higher electron kinetic energy. The low-energy feature rises approximately 35 fs after the highest energy feature at $2.1 \mathrm{eV}$. This maps the initial bending motion as seen in the calculated dynamics, Fig. 2b, and in previous measurements [4]. At longer delay times it is also clear that the centre of mass of the photoelectron spectrum shifts to lower electron kinetic energies, such that the lifetime of the measured photoelectron features is longer at lower electron kinetic ener- 
gies. To obtain a clearer view of the changes observed at the various electron kinetic energies measured, we plot the integrated intensity over the features highlighted in Fig. 4(a) in Fig. 5. The difference in lifetime is apparent in the plots, as well as the appearance of clear oscillations in intensity that peak at times after time zero. The effect of the oscillations is most prominent in the feature centered around $0.9 \mathrm{eV}$, Fig. 5(b), which has a maximum intensity $\sim 200$ fs after excitation. None of the transients can therefore be fit to a simple exponential decay, but are modulated by at least one oscillating component. We therefore fit the transients to an exponential decay modulated by either one or two damped oscillations, convoluted with the instrument response function, corresponding to the laser pulse cross-correlation [28],

$$
g \otimes\left(A_{0} \exp \left(-\frac{t-t_{0}}{\tau}\right) \times \prod^{n} A_{n} \cos \left(\omega_{n}\left(t-t_{0}\right)+\delta_{n}\right)\right) .
$$

Here $A_{n}$ represent intensity scaling parameters, $t_{0}$ the arrival time of the laser pulse, $\tau$ the exponential lifetime and $\omega$ and $\delta$ the angular frequency and phase of the oscillatory component. Fits are plotted as solid lines in Fig. 5. The highest energy feature, Fig. 5(d), provides the clearest data set and contains a single oscillation of period $\sim 0.9 \mathrm{ps}, 38 \mathrm{~cm}^{-1}$, as has previously been experimentally observed $[9,10]$. This corresponds to the beat between the $\nu_{1}$ and $\nu_{2}$ vibrational modes [8]. This is present in each of the other features in the spectrum, along with a second beat with a period around 200 fs. The effect of this oscillation is clearest in the trace presented in Fig. 5(b), however the mixing with the other oscillation and relatively low contrast makes assigning the absolute value of this oscillation difficult, leading to significant error margins. Nonetheless we extract an oscillation period of $220 \mathrm{fs}$, corresponding to $149 \mathrm{~cm}^{-1}$, from this data. While this oscillation period does not fit with any of the known vibrational periods of the molecule, similar frequencies were also observed in a previous study [4] but were not discussed or assigned. The fits to the experimental data furthermore yield an increase in lifetime towards the lower electron kinetic energy regions. The 1/e lifetimes extracted are $401 \mathrm{fs}, 452 \mathrm{fs}$ and $457 \mathrm{fs}$ for the peaks at $2.1 \mathrm{eV}$, $1.4 \mathrm{eV}$ and $0.9 \mathrm{eV}$ respectively.

\section{Discussion}

We now provide a comparison of the experimental measurements and the theoretical calculations. For both the calculations and experiment it is clear 
that the triplet states play a large role in the dynamics from very early times. Significant population is transferred into the triplet states very rapidly with over $50 \%$ of the total population in the triplet states within $250 \mathrm{fs}$. The overall transfer of population approximately matches the decay rate measured in the experiment such that we are only sensitive to the singlet state population. As mentioned above the initial shift in the measured electron kinetic energy maps the initial bending motion of the molecule. As the pump-probe delay increases, the electron kinetic energy shifts towards lower values, such that we observe a longer lifetime for the lower electron kinetic energy regions in the spectrum in Fig. 5. To compare the measured signal to theory, in Fig. 6 we plot the singlet state component of Fig. 2. Initial excitation leads to a wavepacket that oscillates between linear geometries and an angle of $\sim 110^{\circ}$. With increasing pump-probe delay, the range of angles explored narrows and moves away from the linear geometries associated with the spectral feature at the highest electron kinetic energy. Within the current experiment we do not have the time resolution to fully resolve the bending motion, but we do observe the effect of the narrowing and shifting of the angles explored by the molecule as a corresponding narrowing and shifting to lower electron kinetic energies in the photoelectron spectrum.

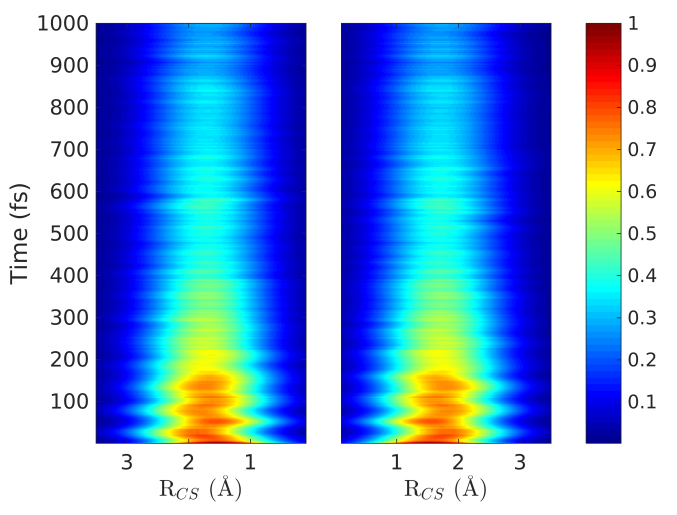

(a) Bond-lengths $R_{\mathrm{CS}}$.

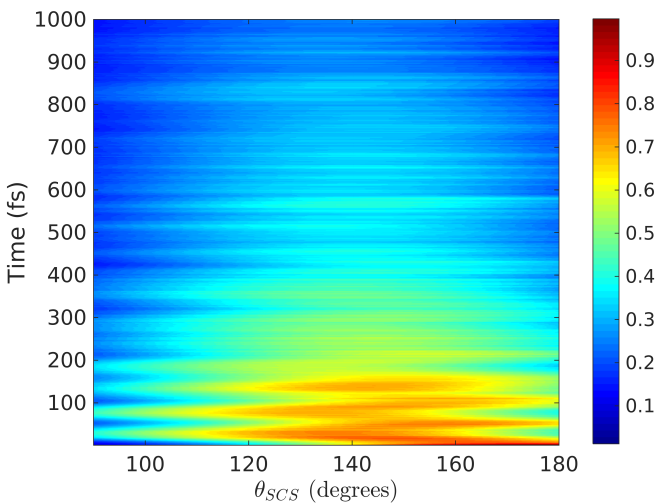

(b) Bending angle $\theta_{\mathrm{SCS}}$.

Figure 6: Probability density evolution of the $\mathrm{CS}_{2}$ geometry in terms of the two bondlengths $R_{\mathrm{CS}}$ and bending angle $\theta_{\mathrm{SCS}}$ in the singlet states only. The intensity bar shows the total population with the decreasing intensity showing the transfer of population into the triplet states.

217 As mentioned above, the short time period oscillation seen both in the 
total photoelectron count rate and, in individual regions of the photoelectron spectrum (Fig. 5), do not match any vibrational periods within the molecule. The simulations also show no obvious changes in the molecular structure that appear to provide an explanation for the oscillations. The calculations do show periodic changes in total singlet excited state population that correlate with the observed changes the photoelectron yield. We therefore tentatively assign the oscillations in the experimental spectrum to changes in the total singlet state population.

\section{Summary}

We have performed a combined theory and experiment study of the excited state dynamics of $\mathrm{CS}_{2}$. The ab-initio surface-hopping simulations highlight the importance of the triplet states in the early time dynamics with significant population transfer predicted, and observed in the complementary time-resolved photoelectron spectroscopy measurements. The combined work demonstrates that one can now do on-the-fly dynamics including spinorbit coupling. The accuracy of the calculation is such that we are able to directly compare the results of the calculation with experiment and explain the shifting and narrowing of the photoelectron spectrum in terms of the bending motion and angles explored by the vibrational wavepacket, while oscillation in the measured photoelectron count rate are explained by the complex coupling of the electronic states that leads to rapid population transfer between manifolds of multiple singlet and triplet excited states. Measuring the longer term dynamics in the triplet states is an experimental challenge. Recent experiments by the Suzuki group using a $7.8 \mathrm{eV}$ probe showed no clear contributions from the triplet states suggesting ionisation requires higher energies. [4] This is presumably due to the ionisation propensity of the triplet states being into electronically excited ion states. Measurements of the dynamics outside of the initially excited singlet states will therefore require measurement using a significantly higher photon energy such as that available from a high harmonic generation source. Such experiments are currently ongoing and will be the subject of a future publication.

\section{Acknowledgments}

All authors thank the STFC for access to the Artemis facility (app. number 13220015). RSM thanks the Royal Society for a University Research 
Fellowship (UF100047) and the Leverhulme trust for research support and for ADS's studentship (RPG-2013-365). HMW thanks the Central Laser Facility and Chemistry at the University of Southampton for a studentship. EJ thanks Chemistry at the University of Southampton for a studentship. We also acknowledge funding from the EC's Seventh Framework Programme (LASERLAB-EUROPE, grant agreement $\mathrm{n}^{\circ} 228334$ ). We thank Phil Rice for technical assistance. This work has been supported by the excellence cluster "The Hamburg Center for Ultrafast Imaging - Structure, Dynamics and Control of Matter at the Atomic Scale" of the Deutsche Forschungsgemeinschaft (CUI, DFG-EXC1074). D.A.H. was supported by the European Research Council through the Consolidator Grant Küpper-614507-COMOTION. AK acknowledges funding from the European Union (FP7-PEOPLE-2013-CIGNEWLIGHT) and the Leverhulme Trust (RPG-2013-365), and DB acknowledges a PhD studentship from the University of Edinburgh. The computational work reported used the ARCHER UK National Supercomputing Service (http://www.archer.ac.uk) and the Edinburgh Compute and Data Facility (ECDF) (http://www.ecdf.ed.ac.uk). DB thanks Sebastian Mai (Wien) for helpful discussions.

[1] D. Townsend, H. Satzger, T. Ejdrup, A. M. D. Lee, H. Stapelfeldt, A. Stolow, ${ }^{1} \mathrm{~b}_{2}$ excited state decay dynamics in $\mathrm{cs}_{2}$, , J. Chem. Phys. 125 (2006) 234302.

[2] T. N. Kitsopoulos, C. R. Gebhardt, T. P. Rakitzis, Photodissociation study of $\mathrm{cs}_{2}$ at $193 \mathrm{~nm}$ using slice imaging, J. Chem. Phys. 115 (2001) 9727.

[3] M. Brouard, E. K. Campbell, R. Cireasa, A. J. Johnsen, W.-H. Yuen, The ultraviolet photodissociation of $\mathrm{cs}_{2}$ : The $\mathrm{s}\left({ }^{1} \mathrm{~d}_{2}\right)$ channel, J. Chem. Phys. 136 (2012) 044310.

[4] R. Spesyvtsev, T. Horio, Y.-I. Suzuki, T. Suzuki, Observation of the wavepacket dynamics on the ${ }^{1} \mathrm{~b}_{2}\left({ }^{1} \sigma_{u}^{+}\right)$state of $\mathrm{cs}_{2}$ by sub-20 fs photoelectron imaging using $159 \mathrm{~nm}$ probe pulses, The Journal of Chemical Physics 142 (2015).

[5] C. Z. Bisgaard, O. J. Clarkin, G. Wu, A. M. D. Lee, O. Gener, C. C. Hayden, A. Stolow, Time-resolved molecular frame dynamics of fixedin-space cs $_{2}$ molecules, Science 323 (2009) 1464. 
[6] T. Horio, R. Spesyvtsev, T. Suzuki, Simultaneous generation of sub-20 fs deep and vacuum ultraviolet pulses in a single filamentation cell and application to time-resolved photoelectron imaging, Opt. Express 21 (2013) 22423.

[7] T. Horio, R. Spesyvtsev, T. Suzuki, Generation of sub-17fs vacuum ultraviolet pulses at $133 \mathrm{~nm}$ using cascaded four-wave mixing through filamentation in ne, Opt. Lett. 39 (2014) 6021.

[8] R. J. Hemley, D. G. Leopold, J. L. Roebber, V. Vaida, The direct ultraviolet absorption spectrum of the ${ }^{1} \sigma_{g}^{+} \rightarrow{ }^{1} \mathrm{~b}_{2}\left({ }^{1} \sigma_{u}^{+}\right)$transition of jetcooled cs2, J. Chem. Phys. 79 (1983) 5219.

[9] P. Farmanara, V. Stert, W. Radloff, Ultrafast predissociation and coherent phenomena in CS2 excited by femtosecond laser pulses at 194-207 nm, The Journal of Chemical Physics 111 (1999) 5338-5343.

[10] P. Hockett, C. Z. Bisgaard, O. J. Clarkin, A. Stolow, Time-resolved imaging of purely valence-electron dynamics during a chemical reaction, Nat Phys 7 (2011) 612-615.

[11] I. M. Waller, J. W. Hepburn, Photofragment spectroscopy of $\mathrm{cs}_{2}$ at 193 nm: Direct resolution of singlet and triplet channels, J. Chem. Phys. 87 (1987) 3261.

[12] D. Xu, J. Huang, W. M. Jackson, Reinvestigation of $\mathrm{cs}_{2}$ dissociation at $193 \mathrm{~nm}$ by means of product state-selective vacuum ultraviolet laser ionization and velocity imaging, J. Chem. Phys. 120 (2004) 3051.

[13] K. Wang, V. McKoy, P. Hockett, M. S. Schuurman, Time-Resolved Photoelectron Spectra of $\mathrm{CS}_{2}$ : Dynamics at Conical Intersections, Phys. Rev. Lett. 112 (2014) 113007.

[14] H.-J. Werner, P. J. Knowles, G. Knizia, F. R. Manby, M. Schütz, et al., Molpro, version 2015.1, a package of ab initio programs, 2015. See.

[15] D. Tseng, R. Poshusta, Ab initio potential energy curves for low-lying states of carbon disulfide, The Journal of chemical physics 100 (1994) 7481-7486. 
[16] Q. Zhang, P. H. Vaccaro, Ab initio studies of electronically excited carbon disulfide, The Journal of Physical Chemistry 99 (1995) 1799 1813.

[17] S. T. Brown, T. J. Van Huis, B. C. Hoffman, H. F. Schaefer III, Excited electronic states of carbon disulphide, Molecular Physics 96 (1999) 693704 .

[18] K. B. Wiberg, Y.-g. Wang, A. E. de Oliveira, S. A. Perera, P. H. Vaccaro, Comparison of cis-and eom-ccsd-calculated adiabatic excited-state structures. changes in charge density on going to adiabatic excited states, The Journal of Physical Chemistry A 109 (2005) 466-477.

[19] A. Mank, C. Starrs, M. Jego, J. Hepburn, A detailed study of the predissociation dynamics of the $1 \mathrm{~b} 2(1 \sigma+\mathrm{u})$ state of cs2, The Journal of chemical physics 104 (1996) 3609-3619.

[20] S. Mai, M. Richter, M. Ruckenbauer, M. Oppel, P. Marquetand, L. González, Sharc: Surface hopping including arbitrary couplings program package for non-adiabatic dynamics, sharc-md.org, 2014.

[21] M. Richter, P. Marquetand, J. González-Vázquez, I. Sola, L. González, SHARC: ab initio molecular dynamics with surface hopping in the adiabatic representation including arbitrary couplings, J. Chem. Theory Comput. 7 (2011) 1253-1258.

[22] S. Mai, P. Marquetand, L. González, A general method to describe intersystem crossing dynamics in trajectory surface hopping, Int. J. Quantum Chem. 115 (2015) 1215-1231.

[23] J. C. Tully, Molecular dynamics with electronic transitions, The Journal of Chemical Physics 93 (1990) 1061-1071.

[24] A. D. Smith, H. M. Watts, E. Jager, D. A. Horke, E. Springate, O. Alexander, C. Cacho, R. T. Chapman, R. S. Minns, Resonant multiphoton ionisation probe of the photodissociation dynamics of ammonia, Phys. Chem. Chem. Phys. 18 (2016) 28150-28156.

[25] A. Eppink, D. Parker, Velocity map imaging of ions and electrons using electrostatic lenses: Application in photoelectron and photofragment ion 
$347 \quad$ imaging of molecular oxygen, Review of Scientific Instruments 68 (1997) $348 \quad 3477-3484$.

[26] D. Irimia, D. Dobrikov, R. Kortekaas, H. Voet, D. A. van den Ende, W. A. Groen, M. H. M. Janssen, A short pulse (7 mu s FWHM) and high repetition rate $(\mathrm{dc}-5 \mathrm{kHz})$ cantilever piezovalve for pulsed atomic and molecular beams, Review of Scientific Instruments 80 (2009) 113303.

[27] G. M. Roberts, J. L. Nixon, J. Lecointre, E. Wrede, J. R. R. Verlet, Toward real-time charged-particle image reconstruction using polar onionpeeling, Review of Scientific Instruments 80 (2009) 053104.

[28] D. Hanggi, P. W. Carr, Errors in exponentially modified Gaussian equations in the literature, Analytical Chemistry 57 (1985) 2394-2395. 